\title{
Artificial Intelligence in Pilot Training and Education - Towards a Machine Learning Aided Instructor Assistant for Flight Simulators
}

\author{
Shuiqiao Yang ${ }^{1[0000-0002-6772-6805]}$, Kun Yu ${ }^{1[0000-0001-5138-6749]}$, Thorsten Lammers ${ }^{2[0000-}$ \\ 0003-1000-2395] and Fang Chen 10000-0003-4971-8729]. \\ ${ }^{1}$ Data Science Institute, University of Technology Sydney, Australia \\ ${ }^{2}$ Centre for Advanced Manufacturing, University of Technology Sydney, Australia \\ shuiqiao.yang@uts.edu.au.com
}

\begin{abstract}
The a viation industry was set to see unprecedented growth over the next two decades. Key occupations predicted to be in shortage included not only pilots, but also flight instructors. Undoubtedly, Covid-19 is currently having a huge impact on the industry. Nevertheless, the current environment further strengthens the need for pilots to maintain their training. Consequently, there is pressure to deliver high-quality training outcomes for an increasing number of pilots and trainees with limited resources available. Current simulator-based training schemes are limited by placing a significant reliance on the personal experience of flight instructors to assess pilot performance. Finding ways to increase the quality and efficiency of simulator-based training is therefore of high importance. With recent advances in artificial intelligence, it is possible to use machine learning techniques to extract latent patterns from massive datasets, to analyze pilot trainees' activities, and to provide feedback on their performance by processing hundreds of different parameters available on flight simulators. An ML-aided pilot training and education framework is needed that exploits the power of the ML techniques for more objective performance evaluation. In this paper, we describe a conceptual framework for such a system and outline steps toward the development of a full digital instructor system with the potential to overcome current limitations and enabling comprehensive and meaningful feedback that is tailored to the individual need of the trainee.
\end{abstract}

Keywords: Aviation Industry, Conceptual Framework, Flight Maneuver

\section{Introduction}

As a special form of education, pilot training is a critical component for the aviation industry and closely relates to flight safety and flight task execution. Well-trained, highly proficient and professional pilots are extremely important for the safety of humans and airplanes [1]. The training process establishes a primary interface between pilots and the potential environment that they will face during real flight operations. Currently, most of the pilot training processes are conducted in a full flight simulator which stimulates actual flight environments and makes the pilot training affordable and safe. For instance, a full flight simulator can simulate accurate force feedback for the 
pilot's flight controls through the simulator system [2]. Equipped with various systems to simulate all the different flight status parameters (e.g. acceleration, altitude, velocity etc.), the flight simulator can generate a large amount of flight and pilot activity data during the training process [3].

With an ability to process large scale datasets and automatically extract hidden features that are difficult to be identified by human beings, machine learning (ML) techniques have developed rapidly in recent years and have been applied in many real-world applications such as computer vision, speech recognition, natural language processing, robotics and medical diagnosis [4]. Overall, ML is capable of processing complex datasets, provide quantitative feedback, extract hidden features, and automatically make decisions.

In this paper, we propose a conceptual framework that utilizes supervised ML techniques to extract the latent patterns from the multimodal data generated by the flight simulator, to build models to characterize the flight tasks, and to recognize the pilot trainees' operational behaviors during the training processes. This framework first trains a supervised ML model to learn the standard pilot operations for the training task using the data generated by experts or teachers. Then, the trained model will be used to provide feedback to pilot trainees during the training process. Overall, the goal of this paper is to propose a framework that utilizes ML algorithms to examine trainee performance in a simulated environment and provide qualitative performance information to aid instructor assessment and enable more effective feedback to trainees in real time.

The remainder of this paper is organized as follows. We first provide the technical background of current pilot training schemes. Then, we review related academic works of ML-related applications in the aviation industry. Afterwards we describe the proposed conceptual ML-aided pilot training framework before concluding with a short summary.

\section{Technical Background}

Flight simulation has been adopted as an integral element in the development of the aviation industry and has contributed significantly to flight safety over the last few decades [5]. It has become an essential means for both civil airlines and military organizations to safely train pilots at an affordable cost. Currently, with the ongoing development of hardware and software technologies, flight simulators are becoming ever more sophisticated to provide training for a wide range of tasks [6]. Fig. 1 shows a flight simulator for a modern civil airline, in which the cabin includes a full replica of the actual flight deck [7]. It includes different subsystems, such as pilot controls, visual, sound and motion, to provide high fidelity cues to the pilots. Additionally, there is a data acquisition system which captures pilot control inputs and simulator state information at a sampling rate (typically $50-60 \mathrm{~Hz}$ ) to record hundreds of inputs and simulator states. This data can be used for pilot trainee performance analysis [6]. 


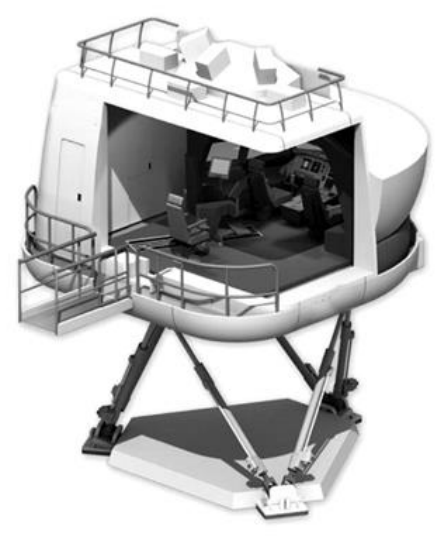

Fig. 1. Illustration of a civil full flight simulator

The instructor station is located at the rear of the cabin, enabling the instructor to observe the operational behaviors of the pilot trainee. In a typical flight training process, the pilot trainee will be accompanied by an instructor for learning and performance evaluation. The flight instructor usually sits behind the trainee to observe the trainee's actions [7]. The flight instructor can introduce simulations of faults, monitor the flight situation, and observe the pilot trainee's control actions [8]. When the training processes are finished, the flight instructor will prepare a debrief report for the trainee in a limited time frame. The report includes the judgement of instructor for the various control actions of the pilot [8]. However, a few challenges have been identified for this instructor-based performance assessment [7] [8]:

- High possibility for an instructor to miss relevant actions of the trainee pilot due to the limited visibility and possible sight obstruction of flight instructor and high instructor workload in boost moments.

- Trainee evaluation is heavily based on the individual experience of instructors. Therefore, the feedback from the instructors is highly subjective and lacks evidence.

- Limited real-time identification and feedback of incorrect pilot activities or risky behaviors during the training process. Very limited retrospective analysis is available to identify the missed performance aspects for pilot trainees.

Modern flight simulators incorporate hundreds of different parameters capturing every aspect of the flight status, such as altitude, velocity, pedal inputs, etc. [6, 9]. The pilot training process leads to a huge amount of data being generated. Identification of the latent operational patterns for pilot trainees can help understand their operational deficiencies quantitatively and objectively, which marks a great difference from the traditional instructor-based subjective feedback. By combining pilot training related data from a flight simulator and advanced ML techniques, it seems promising to develop an intelligent digital instructor system that can provide real-time, objective and quantitative assessments, as well as a detailed diagnosis of the practice processes to the pilot trainees. 


\section{Related Work}

Although the application of ML in the context of flight simulation is a very young field of research, some key contributions have been made. For example, Giddings et al. [10] have studied the problem of defining the relationships between the pre-aptitude, proficiency tests, and success in pilot training. They have collected data with labels from the Air Force Personnel Center and applied different supervised ML models such as random forest, K-nearest neighbors and neural networks to predict the success patterns for pilots based on their historical testing records. Wilson et al. [11] have studied the problem of gaze classification to evaluate the performance for aviators to analyze if the pilot trainee would scan too rapidly, omit, or fixate - which are common errors when scanning the horizon, and cross-checking instruments. Traditionally, the examination for the gaze of pilot trainees relies on a manual check from flight instructors. They have proposed to use ML to replace the flight instructors for this task. They transform the gaze or scan patterns into heatmaps and adopt deep convolutional neural networks to classify the heatmaps into different level of qualities to help the flight instructors for quick reviewing. Memarzadeh et al. [12] have proposed to detect the outliers in flight data using convolutional variational auto-encoders. They argue that the current stateof-the-art ML models show great ability in processing high-dimensional time-series data (like the sequential, time-stamped data generated by a flight simulator). Shmelova et al. [13] have proposed to use ML and text analysis to train the air traffic controllers to decrease human factor-related accident rates. $\mathrm{Li}$ et al. [14] have explored the factors in flight simulators that could improve the human-machine interaction efficiency. They have adopted metacognitive strategy to help pilots to coordinate cognitive resources and gradually form flight experience based on their characteristics.

While these presented works provide insightful analyses of singular aspects within the pilot training process, our proposed conceptual framework focusses on a more comprehensive analysis based on a variety of aspects such as various control inputs to the simulator for the pilot trainees and also aims at facilitating the judgement for flight instructors due to the various challenges mentioned in Section 2.

\section{Conceptual Model}

In order to exploit the potential of ML for pilot training, we propose to utilize supervised $\mathrm{ML}$ algorithms to learn the latent reverse mapping functions between the flight status and the corresponding operational inputs in a maneuver task.

A flight simulator records two types of data: one type for capturing the simulated aircraft states and the other for capturing the operator's control actions. The control actions performed by the trainee lead to the corresponding changes of the flight states such as attitude, altitude and airspeed. It is nontrivial to explicitly define the mapping functions given the large amount of control inputs and state variables, but supervised ML algorithms could easily capture the relations between them by using the status signals as training data and the control signals as the corresponding label information. It 
is intuitive the actions of pilots with different proficiency levels would result in different simulated aircraft states and the associated control actions when conducting the same maneuver task.

To create such training and labelling data pairs, we assume that the expert pilots can perform standard control operations and their generated data from the flight simulators can accurately reflect the mapping relations between flight status and control actions. Hence, it is possible for us to train the supervised ML algorithms using the training datasets collected from experts. After the supervised ML model has been trained, those datasets can be used to predict the expert-level control signals given the current flight status performed by pilot trainees, thus differences and feedbacks could be provided to the trainees in real time.

The proposed framework utilizes supervised ML techniques as a novel tool to assist pilot training. As shown in Fig. 2, it involves two core modules: an offline module to use the expert pilots' behavior data to train supervised ML models; and an online module to provide the trainee pilot with feedback.

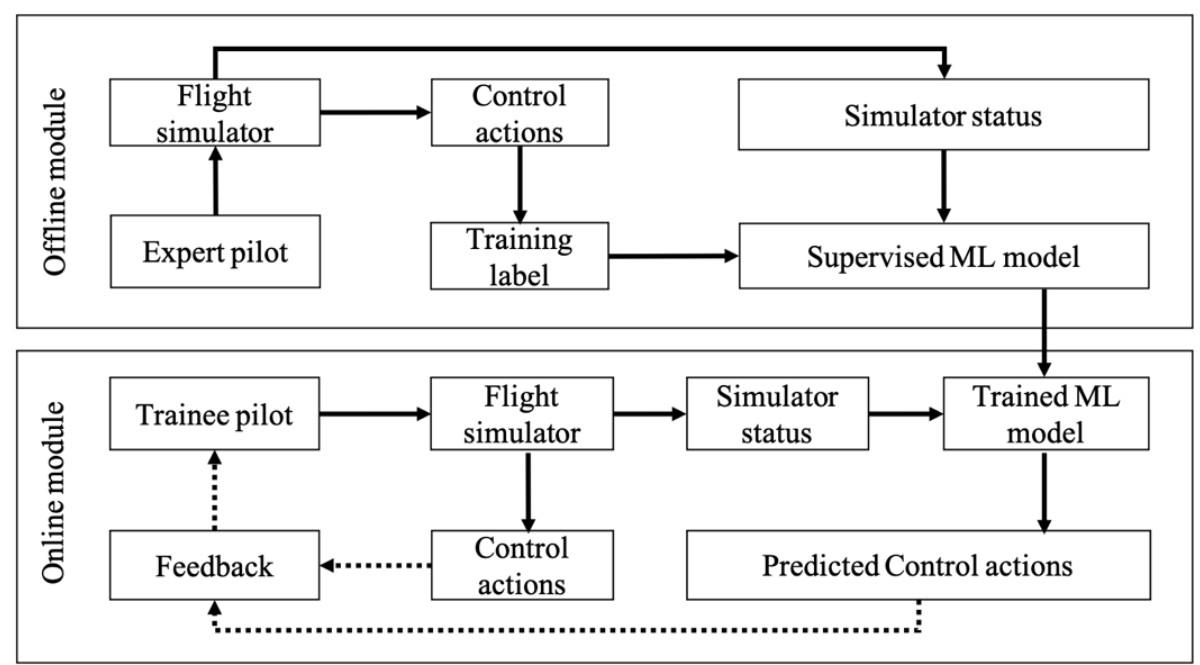

Fig. 2. Conceptual framework for ML-aided pilot training and education (the solid arrow lines denote the data flows and the dotted arrow lines denote the feedbacks)

\subsection{Offline module for standard pilot operation learning}

The offline module contains supervised ML models that are trained with labelled data to learn the standard operations for different maneuvers. The purpose of the offline module is to train the supervised ML model to learn the expert-level control actions for a maneuver task. As seen in Fig. 2, we assume that the operations from expert pilots for a maneuver are standardized and could be used to create a training dataset. Hence, we propose to allow the expert pilot to perform the standard operations for a maneuver and the data collected from the simulator are used as training dataset for the ML model. To train the ML model for recognizing the standard control actions on a specific maneuver, 
we can use the control actions data as the training target (i.e., labels) and the simulated aircraft state data as the training input. In this way, the trained ML model can be used to predict expert-level control actions given the flight status performed by trainees. After the ML model has been trained for a specific maneuver, it can be used for providing feedback to pilot trainees in their training processes when they perform the same maneuver task.

\subsection{Online module for providing real time feedback}

The online module shown in Fig. 2 is designed for providing comprehensive real-time feedback to pilot trainees. In the traditional pilot training process, the instructors have limited time to prepare the debrief report to the trainees and the feedback from the instructors can also be subjective. Using the online module, the real-time data collected from the simulator will be separated into two parts: the flight status data and the control action data. The trained ML models are able to learn the relations between the flight status and control actions. When given the state data of the simulator operated by a pilot trainee, the differences between the expert-level control actions and the current pilot trainee's control actions can be computed. Thus, detailed feedback can be provided to the trainee in real time.

Based on the new ML-aided pilot training framework, the pilot trainees are expected to gain a better understanding of their control behaviors as the control difference between the trainee and the expert can be provided as feedback in real-time. Also, for each control input to the simulator, the pilot trainee can view the difference between themselves and the expert. Therefore, the ML models can provide more comprehensive and detailed feedback to pilot trainee. At the same time, the flight instructor may also have access to more evidence supporting them for preparing the debrief report.

\section{Conclusion}

Pilot training has always been an essential element of the aviation industry. Welltrained pilots are essential for increasing the safety of various flight activities. In this paper, we review the current pilot training practices that are based on the feedback from flight instructors and explain the drawbacks of the current instr uctor-based evaluation system. Based on the rapid progress in the development of ML techniques, we propose a framework that exploits the power of ML methods for better pilot training. In the framework, the standard operational patterns for different maneuvers are learned by the supervised ML models. After that, the pilot trainee can be provided with real-time feedback in a more comprehensive way, utilizing the prediction capabilities of trained ML models. We believe that our proposed framework provides a starting point for a development initiative and can also be generalized to other professional training and education contexts such as medical surgery training, operation of precision instruments, etc. In the future, we will exploit the proposed conceptual framework into the real flight simulators and develop a reliable digital assistant for pilot training. 


\section{References}

1. Orlady, L.M., Airline pilot training today and tomorrow, in Crew resource management. 2010, Elsevier. p. 469-491.

2. $\quad$ Feng, T. and C.-q. Zhao, Design and Research of Electric Control Load System of Flight Simulator. DEStech Transactions on Engineering and Technology Research, 2016(iceea).

3. Mangortey, E., et al. Application of Machine Learning Techniques to Parameter Selection for Flight Risk Identification. in AIAA Scitech 2020 Forum. 2020.

4. $\quad$ LeCun, Y., Y. Bengio, and G. Hinton, Deep learning. nature, 2015. 521(7553): p. 436-444.

5. Baarspul, M., A review of flight simulation techniques. Progress in Aerospace Sciences, 1990. 27(1): p. 1-120.

6. Boril, J., M. Jirgl, and R. Jalovecky, Using Aviation Simulation Technologies for Pilot Modelling and Flight Training Assessment. Advances in Military Technology, 2017. 12(1).

7. Allerton, D., The impact of flight simulation in aerospace. The Aeronautical Journal, 2010. 114(1162): p. 747-756.

8. Hays, R.T., et al., Flight simulator training effectiveness: A meta-analysis. Military psychology, 1992. 4(2): p. 63-74.

9. Boril, J., M. Jirgl, and R. Jalovecky. Use of flight simulators in analyzing pilot behavior. in IFIP International Conference on Artificial Intelligence Applications and Innovations. 2016. Springer.

10. Giddings, A.C., Predicting Pilot Success Using Machine Learning. 2020.

11. Wilson, J., et al., Automatic Gaze Classification for Aviators: Using Multi-task Convolutional Networks as a Proxy for Flight Instructor Observation. International Journal of Aviation, Aeronautics, and Aerospace, 2020. 7(3): p. 7.

12. Memarzadeh, M., B. Matthews, and I. Avrekh, Unsupervised Anomaly Detection in Flight Data Using Convolutional Variational Auto-Encoder. Aerospace, 2020. 7(8): p. 115.

13. Shmelova, T., et al., Machine Learning and Text Analysis in an Artificial Intelligent System for the Training of Air Traffic Controllers, in Automated Systems in the Aviation and Aerospace Industries. 2019, IGI Global. p. 1-50.

14. Li, Q., et al. Human-Machine Interaction Efficiency Factors in Flight Simulator Training Towards Chinese Pilots. in International Conference on Applied Human Factors and Ergonomics. 2020. Springer. 